\title{
A HOMOMORPHISM THEOREM FOR MULTIPLIERS
}

\author{
by NAKHLÉ HABIB ASMAR
}

(Received 18th August 1987)

\section{Notation and introduction}

Throughout the paper, the symbols $G_{1}$ and $G_{2}$ will denote two locally compact abelian groups with character groups $X_{1}$ and $X_{2}$, respectively. Haar measures on $G_{j}$ are denoted by $\mu_{j}$; the ones on $X_{j}$ are denoted by $\theta_{j}(j=1,2)$. The measures $\mu_{j}$ and $\theta_{j}$ are normalized so that the Plancherel Theorem holds (see [7, p. 226, Theorem 31.18]).

If $G$ is a locally compact abelian group with character group $X$, and if $f$ is a complex-valued function on $G$, then $f$ is said to be measurable means that $f$ is measurable with respect to Haar measure on $G$. The class of measurable functions on $G$, with integrable $p$ th power, is denoted by $\mathscr{L}_{p}(G) 1 \leqq p<\infty$; the class of essentially bounded measurable functions by $\mathscr{L}_{\infty}(G)$; the class of continuous functions with compact support by $\mathscr{C}_{\infty}(G)$.

If $A$ is a subset of $G$, the complement of $A$ in $G$ is denoted by $A^{\prime}$ or $G \backslash A$. The symbol $1_{A}$ will denote the indicator function of the set $A$. All other notation used in this paper without explanation is as in [6] and [7]. A bounded measurable function $m$ on $X$ is called an $\mathscr{L}_{p}(G)$-multiplier, $1 \leqq p<\infty$, if for every $f$ in $\mathscr{L}_{p}(G) \cap \mathscr{L}_{2}(G)$ there is a $g$ in $\mathscr{L}_{p}(G)$ such that $\hat{g}=m \hat{f}$, and $\|g\|_{p} \leqq N_{p}(m)\|f\|_{p}$, where $N_{p}(m)$ is the norm of the unique extension of the bounded linear operator $f \rightarrow g$ to all of $\mathscr{L}_{p}(G)$. We shall denote this extension by $T_{m}$. The set of all multipliers on $\mathscr{L}_{p}(G)$ will be denoted by $M_{p}(G)$.

Suppose that $\tau$ is a continuous nonzero homomorphism from $X_{2}$ into $X_{1}$. A well-known theorem for multipliers asserts that if $m$ is continuous on $X_{1}$, then $m \circ \tau$ is in $M_{p}\left(G_{2}\right)$ and $N_{p}(m \circ \tau) \leqq N_{p}(m)$. (See [5, Theorem B.2.1, p. 187]). We will refer to this fact as the homomorphism theorem for continuous multipliers.

Many interesting multipliers are not continuous; e.g. the sgn function on $\mathbb{R}$ which is an $\mathscr{L}_{p}(\mathbb{R})$-multiplier for $1<p<\infty$. Our goal, in this essay, is to give a new proof of the homomorphism theorem for continuous multipliers based on the so-called transference methods, then derive a more general version that applies to multipliers like the sgn function.

\section{The homomorphism theorem}

We continue with the notation of Section $1: m$ is a bounded continuous function on $X_{1}$, and $\tau$ is a continuous nonzero homomorphism from $X_{2}$ into $X_{1}$. 
2.1. An approximate unit in $\mathscr{L}_{1}\left(X_{1}\right)$.

By interchanging the group $G$ and its character group $X$ in Theorem 33.12 p. 298 of [7], we see that $\mathscr{L}_{1}\left(X_{1}\right)$ contains a net of functions $\left(\hat{u}_{i}\right)_{1 \in I}$ such that, for all $l$ in $I$, we have:

$$
\begin{gathered}
\hat{u}_{\imath} \geqq 0 ; \\
\int_{X_{1}} \hat{u}_{1} d \theta_{1}=1 ; \\
u_{\imath} \geqq 0 ; \quad \text { and } \quad u_{\imath} \in \mathscr{C}_{\infty}\left(G_{1}\right) .
\end{gathered}
$$

From (1) and (2), it follows that

$$
\lim \hat{u}_{i} * m=m
$$

uniformly on compact subsets of $X_{1}$. (Use (1), (2), and (28.52) of [7]).

Clearly, from (4), we have

$$
\lim _{\mathfrak{l}} \hat{u}_{\imath} * m \circ \tau=m \circ \tau
$$

uniformly on compact subsets of $X_{2}$.

Theorem 2.2. Suppose that $m$ is a bounded and continuous function on $X_{1}$ which is also in $M_{p}\left(G_{1}\right), 1 \leqq p<\infty$. Let $\tau$ be a continuous homomorphism from $X_{2}$ into $X_{1}$. Then $m \circ \tau$ is an $\mathscr{L}_{p}\left(G_{2}\right)$-multiplier with $N_{p}(m \circ \tau) \leqq N_{p}(m)$.

The proof of Theorem 2.2 combines a transference results and well-known properties of translation-invariant operators. We shall start with the transference set-up. Suppose that $k$ is in $\mathscr{L}_{1}\left(G_{1}\right)$ with compact support. Let $T_{k}$ denote the operator $f \mapsto f * k$, and let $N_{p}(k)$ denote its norm as an operator from $\mathscr{L}_{p}\left(G_{1}\right)$ into $\mathscr{L}_{p}\left(G_{1}\right)$. Let $\phi$ denote a continuous nonzero homomorphism from $G_{1}$ into $G_{2}$. If $f$ is in $\mathscr{L}_{p}\left(G_{2}\right)$, using [6, Lemma $20.6, p$. 287], one can easily show that the function $(t, x) \mapsto f(x-\phi(t))$ is measurable with respect to the product measure on $G_{1} \times G_{2}$.

Let $T_{k}^{\#}$ denote the operator, defined in $\mathscr{L}_{p}\left(G_{2}\right)$ by

$$
T_{k}^{\#} f(x)=\int_{G_{1}} f(x-\phi(t)) k(t) d \mu_{1}(t)
$$

Applying Theorem 2.4 of [4], we see that the inequality

$$
\left\|T_{k}^{\#} f\right\|_{p} \leqq N_{p}(k)\|f\|_{p}
$$

holds for all $f$ in $\mathscr{L}_{p}\left(G_{2}\right)$ with $1 \leqq p<\infty$. (While in [4] it is required that $G_{2}$ be 
$\sigma$-compact, one can check that the proof of [4, Theorem 2.4], still holds when $G_{2}$ is not $\sigma$-compact and the operator is of the particular form (6). See also Theorem 2.3 of [3]).

Lemma 2.3. Let $m$ and $u_{1}$ be as in (2.1). Let $h$ be in $\mathscr{L}_{2}\left(G_{1}\right) \cap \mathscr{C}_{\infty}\left(G_{1}\right)$ such that $\hat{h}$ is in $\mathscr{L}_{1}\left(X_{1}\right)$. Set

$$
k_{i}=\left(\left(\hat{u}_{i} * m\right) \hat{h}\right)^{\mathrm{v}} .
$$

Then $k_{1}$ is in $\mathscr{L}_{1}\left(G_{1}\right)$ with support contained in $\operatorname{supp} u_{1}+\operatorname{supp} h$. In particular, supp $k_{1}$ is compact.

Proof. We have

$$
\begin{aligned}
\left(\left(m * \hat{u}_{1}\right) \hat{h}\right)^{\vee}(x) & =\int_{X_{1}} m * \hat{u}_{i}(\gamma) \hat{h}(\gamma) \gamma(x) d \theta_{1}(\gamma) \\
& =\int_{x_{1}} \int_{X_{1}} m(\eta) \hat{u}_{i}(\gamma-\eta) d \theta_{1}(\eta) \hat{h}(\gamma) \gamma(x) d \theta_{1}(\gamma) \\
& =\int_{X_{1}} m(\eta) \int_{X_{1}} \hat{u}_{l}(\gamma-\eta) \hat{h}(\gamma) \gamma(x) d \theta_{1}(\gamma) d \theta_{1}(\eta) \\
& =\int_{X_{1}} m(\eta) h *\left(\eta u_{1}\right)(x) d \theta_{1}(\eta) .
\end{aligned}
$$

Note that $\operatorname{supp} \eta u_{1} \subseteq \operatorname{supp} u_{1}$. Thus,

$$
\operatorname{supp}\left(h *\left(\eta u_{i}\right)\right) \subseteq \operatorname{supp} h+\operatorname{supp} u_{i}
$$

from which the lemma follows.

Lemma 2.4. Suppose that $m$ is in $M_{p}\left(X_{1}\right)$ ( $m$ need not be continuous). With the notation of Lemma 2.3 , we have

(a) $N_{p}\left(u_{1} * m\right) \leqq N_{p}(m)$,

(b) $\left\|k_{1} * f\right\|_{p} \leqq N_{p}(m)\|h\|_{1}\|f\|_{p}$

for all $\iota \in I$, and all $f$ in $\mathscr{L}_{p}\left(G_{1}\right), 1 \leqq p<\infty$.

Proof. Part (a) is a well-known property of multipliers. For its proof see [5, B.1.2. (iii), p. 185].

For (b), it is enough to consider $f$ in $\mathscr{L}_{p}(G)$ with compactly supported $\hat{f}$. We have

$$
\left\|f *\left(\left(\hat{u}_{1} * m\right) \hat{h}\right)^{\vee}\right\|_{p}=\| h *\left(\hat{f}^{\left.\left(\hat{u}_{1} * m\right)\right)^{\vee} \|_{p}}\right.
$$




$$
\begin{aligned}
& \left.\leqq\|h\|_{1} \| \hat{f}\left(\hat{u}_{1} * m\right)\right)^{\vee} \|_{p} \\
& \leqq N_{p}(m)\|h\|_{1}\|f\|_{p} \quad \text { (from (a)). }
\end{aligned}
$$

\section{5.}

We now go back to our set-up of (2.1). We have a continuous homomorphism $\tau$ from $X_{2}$ into $X_{1}$. To use the transference results, we introduce the adjoint homomorphism $\phi$ of $\tau$; thus $\phi$ is the continuous homomorphism from $G_{1}$ into $G_{2}$ satisfying the identity

$$
\chi \circ \phi(s)=\tau(\chi)(s)
$$

for all $\chi$ in $X_{2}$, and all $s$ in $G_{1}$.

For every $\imath \in I$ and $f \in \mathscr{L}_{p}\left(G_{2}\right), 1 \leqq p<\infty$, we let

$$
T_{k_{1}}^{\#} f(x)=\int_{G_{1}} f(x-\phi(t)) k_{1}(t) d \mu_{1}(t)
$$

where $k_{t}=\left(\left(m * \hat{u}_{1}\right) \hat{h}\right)^{\vee}, h$ is an arbitrary but fixed element in $\mathscr{L}_{2}\left(G_{1}\right) \cap \mathscr{C}_{\infty}\left(G_{1}\right)$ such that $\|h\|_{1} \leqq 1$, and $\bar{h}$ is an $\mathscr{L}_{1}\left(X_{1}\right)$.

Using (2.2.7) and (2.4.b) we see that

$$
\left\|T_{k_{2}}^{\#} f\right\|_{p} \leqq N_{p}(m)\|f\|_{p}
$$

for all $f$ in $\mathscr{L}_{p}\left(G_{2}\right)$.

Lemma 2.6. Notation is as in (2.5). Let $f$ be in $\mathscr{L}_{p}\left(G_{2}\right) \cap \mathscr{L}_{1}\left(G_{2}\right), 1 \leqq p<\infty$. We have (a)

$$
\left(T_{k_{1}}^{\#} f\right) \hat{(}(\chi)=\hat{f}(\chi) \hat{u}_{1} * m(\tau(\chi)) \hat{h}(\tau(\chi))
$$

for all $\chi$ in $X_{2}$ and all $\iota \in I$;

$$
\lim \left(T_{k_{1}}^{*} f\right) \hat{(}(\chi)=\hat{f}(\chi) m(\tau(\chi)) \hat{h}(\tau(\chi))
$$

uniformly on compact subsets of $X_{2}$.

Proof. We have

$$
\begin{aligned}
\left(T^{\#} f\right) \hat{(\chi)} & =\int_{G_{2}} \bar{\chi}(x) \int_{G_{1}} f(x-\phi(t)) k_{1}(t) d \mu_{1}(t) d \mu_{2}(x) \\
& =\int_{G_{1}} \int_{G_{2}} \bar{\chi}(x) f(x-\phi(t)) d \mu_{2}(x) k_{1}(t) d \mu_{1}(t)
\end{aligned}
$$




$$
\begin{aligned}
& =\int_{G_{1}} \int_{G_{2}} \bar{\chi}(x+\phi(t)) f(x) d \mu_{2}(x) k_{1}(t) d \mu_{1}(t) \\
& =\hat{f}(\chi) \int_{G_{1}} \bar{\chi}(\phi(t)) k_{t}(t) d \mu_{1}(t) \\
& =\hat{f}(\chi) \int_{G_{1}} \overline{\tau(\chi)}(t) k_{t}(t) d \mu_{1}(t) \\
& =\hat{f}(\chi) \hat{k}_{l}(\tau(\chi)) \\
& =\hat{f}(\chi) u_{1} * m(\tau(\chi)) \hat{h}(\tau(\chi)) .
\end{aligned}
$$

Part (b) is an immediate consequence of (a) and (2.1.5).

2.7 Proof of Theorem 2.2. Let $1 \leqq p<\infty$, and let $q=(p / p-1)$ if $1<p<\infty$, and $q=\infty$ if $p=1$. It is enough to show that

$$
\left|\int_{G_{2}}(\hat{f}(m \circ \tau))^{\vee}(x) \bar{g}(x) d \mu_{2}(x)\right| \leqq N_{p}(m)\|f\|_{p}\|g\|_{q}
$$

for all $f \in \mathscr{L}_{p}\left(G_{2}\right) \cap \mathscr{L}_{1}\left(G_{2}\right), g \in \mathscr{L}_{q}\left(G_{2}\right) \cap \mathscr{L}_{1}\left(G_{2}\right)$, and $\hat{f}$ and $\hat{g}$ are in $\mathscr{C}_{\infty}\left(X_{2}\right)$. (See [5, 1.2.2. (iii), p. 7]). We have from (2.6b)

$$
\lim _{\imath} \overline{\hat{g}}(\chi) \hat{f}(\chi) \hat{u}_{\imath} * m(\tau(\chi)) \hat{h}(\tau(\chi))=\overline{\hat{g}}(\chi) \hat{f}(\chi) m(\tau(\chi)) \hat{h}(\tau(\chi))
$$

uniformly on $X_{2}$. Also note that the inequality

$$
\left|\hat{f}(\chi) \overline{\hat{g}}(\chi)\left(\hat{u}_{1} * m\right)(\tau(\chi)) \hat{h}(\tau(\chi))\right| \leqq\|\hat{g}\|_{\infty}\|m\|_{\infty}\|\hat{h}\|_{\infty}\|\hat{f}\|_{\infty}
$$

holds for all $\chi$ in $X_{2}$ and all $\iota \in I$. From (10), (11), (2.5.8), and Parseval's identity ([7, 31.19 , p. 226]), we infer that

$$
\begin{aligned}
\left|\int_{X_{2}} \overline{\hat{g}} \hat{\jmath} m \circ \tau \hat{h} \circ \tau d \theta_{2}\right| & =\left|\lim \int_{X_{2}} \overline{\hat{g}} \hat{f}\left(\hat{u}_{1} * m\right) \circ \tau \hat{h} \circ \tau d \theta_{2}\right| \\
& =\lim \left|\int_{G_{2}} \bar{g} T_{k_{i}}^{\#} f d \mu_{2}\right|
\end{aligned}
$$




$$
\leqq N_{p}(m)\|f\|_{p}\|g\|_{q}
$$

We now show that (12) implies (9). Using Parseval's identity, rewrite the left side of (9) as

$$
\left|\int_{X_{2}} \hat{f}(\chi) m \circ \tau(\chi) \overline{\hat{g}}(\chi) d \theta_{2}(\chi)\right|
$$

Denote by $K$ the support of $\hat{f} \hat{g}$. Given $\varepsilon>0$, let $h$ in $\mathscr{L}_{2}\left(G_{1}\right) \cap \mathscr{C}_{\infty}\left(G_{1}\right)$ be such that $\|h\|_{1}=1, h \in \mathscr{L}_{1}\left(X_{1}\right)$, and

$$
|\hat{h}(\chi)-1|<\varepsilon
$$

for all $\chi$ in $\tau(K)$. (To find $h$, use [7, Theorem 33.11 p. 298]). We have

$$
\left|\int_{X_{2}} \hat{f}(\chi) m \circ \tau(\chi) \overline{\hat{g}}(\chi) d \theta_{2}(\chi)-\int_{X_{2}} \hat{f}(\chi) \hat{h}(\tau(\chi)) m \circ \tau(\chi) \overline{\hat{g}}(\chi) d \theta_{2}(\chi)\right|<\varepsilon\|f\|_{\infty}\|g\|_{\infty}\|m\|_{\infty} \theta_{2}(K)
$$

Clearly, this together with (12) implies (9).

2.8. Remark. The assumption 2.1 .5 can be replaced by the requirement that $\left(\hat{u}_{1} * m\right) \circ$ $\tau$ converges to $m \circ \tau$ in the weak-star topology of $\mathscr{L}_{\infty}\left(X_{2}\right)$. For in this case, to establish 2.7.1, we would start with the equality

$$
\left|\int_{X_{2}} \hat{f} \overline{\hat{g}} m \circ \tau \hat{h} \circ \tau d \theta_{2}\right|=\left|\lim \int_{X_{2}} \hat{f} \overline{\hat{g}}\left(u_{1} * m\right) \circ \tau \hat{h} \circ \tau d \theta_{2}\right|,
$$

and then continue the proof 2.7 from 2.7.12 until the end without a hitch.

Our next version of the homomorphism theorem applies to normalized multipliers.

Definition 2.9. A bounded function $m$ on $X$ is said to be normalized if there is an approximate identity $\left(k_{n}\right)_{n=1}^{\infty}$ in $\mathscr{L}_{1}\left(X_{1}\right)$ such that $\lim _{n \rightarrow \infty} k_{n} * m(\chi)$ exists for all $\chi$ in $X$. We denote this limit by $m^{*}$.

Theorem 2.10. Let $m$ be a normalized function in $M_{p}\left(X_{1}\right), 1 \leqq p<\infty$, and let $\tau$ be a continuous homomorphism of $X_{2}$ into $X_{1}$. Then the function $m^{*} \circ \tau$ is in $M_{p}\left(X_{2}\right), 1 \leqq p<\infty$, with $N_{p}\left(m^{*} \circ \tau\right) \leqq N_{p}(m)$. 
Theorem 2.10 is an immediate consequence of Theorem 2.2 and the following lemma whose proof can be reconstructed from [5, pp. 190-191, B.2.2, (i)-(iv)].

Lemma 2.11. Let $X$ be a locally compact abelian group with character group $G$. Let $\left(m_{n}\right)_{n=1}^{\infty}$ be a sequence of continuous functions in $M_{p}(X), 1 \leqq p<\infty$, such that:

$$
\sup _{n}\left\|m_{n}\right\|_{\infty}<\infty
$$

$$
\lim _{n \rightarrow \infty} m_{n}(\chi)=m(\chi)
$$

for all $\chi$ in $X$; and

$$
\sup _{n} N_{p}\left(m_{n}\right)=c_{p}<\infty
$$

Then $m$ is in $M_{p}(X)$ with $N_{p}(m) \leqq c_{p}$.

To prove Theorem 2.10 note that the functions $k_{n} * m \circ \tau$ have the following properties: $k_{n} * m \circ \tau$ are continuous, and

$$
\begin{gathered}
\left\|k_{n} * m \circ \tau\right\|_{\infty} \leqq\left\|k_{n} * m\right\|_{\infty} \\
\leqq\left\|k_{n}\right\|_{1}\|m\|_{\infty}=\|m\|_{\infty} ; \\
\lim _{n \rightarrow \infty} k_{n} * m \circ \tau=m^{*} \circ \tau
\end{gathered}
$$

pointwise everywhere on $X_{2}$; and

$$
\begin{aligned}
N_{p}\left(k_{n} * m \circ \tau\right) & \leqq N_{p}\left(k_{n} * m\right)(\text { by Th. } 2.2) \\
& \leqq N_{p}(m)(\text { by }(2.4)(\mathrm{a})) .
\end{aligned}
$$

Now apply Lemma 2.11 to the sequence $\left(k_{n} * m \circ \tau\right)_{n=1}^{\infty}$ in $M_{p}\left(X_{2}\right)$.

A version of Theorem 2.10 appears in [3, Theorem 2.7]. Its proof, while quite different from ours, also uses the transference methods.

\section{Applications}

An interesting application of Theorem 2.10 to multiple Fourier series is obtained by taking: $X_{1}=\mathbb{T}$ (the unit circle parametrized by the interval $\left[-\pi, \pi\left[; X_{2}=\mathbb{Z}^{n}\right.\right.$ where $n$ is a positive integer; and $m=1_{l a, b[}$ where $-\pi \leqq a<b<\pi$. The homomorphism $\tau$ is given by 


$$
\tau\left(m_{1}, m_{2}, \ldots, m_{n}\right)=\sum_{j=1}^{n} \alpha_{j} m_{j}(\bmod 2 \pi) \text { where }
$$

$\left\{\alpha_{1}, \alpha_{2}, \ldots, \alpha_{n}\right\}$ is a subset of $\mathbb{R}$ which is linearly independent over $\mathbb{Q}$. The case $n=1$ is presented in [8], Section 1.

We now derive a generalization of $\mathrm{M}$. Riesz's theorem on conjugate functions by using the original version on $\mathbb{R}$. This approach to the abstract of $\mathbf{M}$. Riesz's theorem is due to [2] for compact abelian groups, and to [1] for arbitrary locally compact abelian groups.

We take $X_{1}=\mathbb{R}, G_{1}=\mathbb{R}$; and we write $X$ and $G$ for $X_{2}$ and $G_{2}$, and $\mu$ and $\theta$ for $\mu_{2}$ and $\theta_{2}$. We suppose that $X$ contains a measurable subset $P$ such that $P+P=P$; $P \cap(-P)=\{0\} ; P \cup(-P)=X$. Such a set is called an order on $X$. With $P$ we associate the function $\operatorname{sgn}_{P}$ defined on $X$ by

$$
\operatorname{sgn}_{P}(\chi)=\left\{\begin{array}{rll}
1 & \text { if } & \chi \in P \backslash\{0\} \\
0 & \text { if } & \chi=0 \\
-1 & \text { if } & \chi \in(-P) \backslash\{0\} .
\end{array}\right.
$$

An abstract version of $\mathbf{M}$. Riesz's theorem for conjugate function can be stated as follows.

Theorem 3.1. Notation is as above. Let $f$ be in $\mathscr{L}_{p}(G) \cap \mathscr{L}_{2}(G), 1<p<\infty$. We have

$$
\left\|\left(-i \operatorname{sgn}_{P} \hat{f}\right)^{\vee}\right\|_{p} \leqq A_{p}\|f\|_{p}
$$

where the constant $A_{p}$ is the same as the constant appearing in $M$. Riesz's theorem on $\mathbb{R}$ (or T).

Proof. It is enough to consider $f$ in $\mathscr{L}_{p}(G)$ such that $\hat{f}$ is in $\mathscr{C}_{\infty}(X)$.

Let $K$ be the support of $\hat{f}$. Apply Theorem (5.14) of [1] to obtain a homomorphism $\tau$ from $X$ into $\mathbb{R}$ such that the equality

$$
\operatorname{sgn}_{p}(\chi)=\operatorname{sgn}(\tau(\chi))
$$

holds for $\theta$-almost all $\chi$ in $X$. We clearly have

$$
\left(-i \operatorname{sgn}_{P} \hat{f}\right)^{\vee}=(-i \operatorname{sgn} \circ \tau \hat{f})^{\vee}
$$

$\theta$-almost everywhere on $X$.

The function $s \rightarrow-i \operatorname{sgn}(s)$ is normalized on $\mathbb{R}$. M. Riesz's theorem on $\mathbb{R}$ asserts that 
the function $s \mapsto-i \operatorname{sgn}(s)$ is an $\mathscr{L}_{p}(\mathbb{R})$-multiplier with norm $A_{p}$. The inequality (i) follows now from (13) and Theorem 2.10 .

The following results is due to [8, Section 3], for the case $X=\mathbb{R}^{n}$; to [4, (3.16)] for the case $G \sigma$-compact; and to $[9,(4.6)(b)]$, for the general case under more hypothesis than we require below.

Theorem 3.2. Let $m$ be a normalized function on $X$ which is an $\mathscr{L}_{p}(G)$-multiplier with norm $N_{p}(m), 1 \leqq p<\infty$. Suppose further that $m^{*}=m$. Let $Y$ be a closed subgroup of $X$. Suppose that $f$, the restriction of $m$ to $Y$, is measurable with respect to the Haar measure on $Y$. Then $f$ is an $\mathscr{L}_{p}(G / A(G, Y))$-multiplier, where $A(G, Y)=\{g \in G: g(\chi)=1$ for all $\chi$ in $Y$ \}. Moreover, we have $N_{p}(f) \leqq N_{p}(m)$. 2.10.

Proof. Let $\tau$ be the identity homomorphism from $Y$ into $X$. Apply Theorem

\section{REFERENCES}

1. Nakhle Asmar and Edwin Hewitt, Marcel Riesz's theorem on conjugate Fourier series and its descendants, Proceedings of the analysis conference, held in Singapore June 1986 (NorthHolland, to appear).

2. Earl Berkson and T. A. Gillespie, The generalized M. Riesz theorem and transference, Pacific J. Math. 120 (2) (1985), 279-288.

3. Earl Berkson, T. A. Gillespie and Paul Muhly, Generalized analyticity in UMD spaces, Ark. Mat., to appear.

4. Ronald Colfman and Guido Weiss, Transference methods in analysis, Regional conference series in Math. 31 (Amer. Math. Soc., Providence, 1977).

5. R. E. Edwards and G. I. Gaudry, Littlewood-Paley and multiplier theory (Berlin, Heidelberg, New York: Springer-Verlag, 1977).

6. Edwin Hewitt and Kenneth Ross, Abstract Harmonic Analysis I, Second Edition (Berlin, Heidelberg, New York: Springer-Verlag, 1979).

7. Edwin Hewitt and Kenneth Ross, Abstract Harmonic Analysis II, (Berlin, Heidelberg, New York: Springer-Verlag, 1970).

8. Karel Deleeuw, On L $\mathrm{L}_{\mathrm{p}}$ multipliers, Ann. of Math. 81 (1965), 364-379.

9. SADAhIRo SAEKI, Translation invariant operators on groups, Tôhoku Math. J. 22 (1970), 409-419.

Department of Mathematics and Computer Science

California State University, Long Beach

Long, Beach, California 90840

USA 